P0-0567

WITHDRAWN

\section{PO-0568 TRANSIENT HYPERPHOSPHATEMIA IN INFANTS WITH VIRAL INFECTIONS}

${ }^{1} S$ Smugreska, ${ }^{2} \mathrm{~V}$ Tasic, ${ }^{3} \mathrm{M}$ Trenceva, ${ }^{3} \mathrm{~S}$ Todorovska, ${ }^{4} \mathrm{~A}$ Sofijanova. ${ }^{1}$ Paediatric, Private Pediatric Pratctice, Skopje, Macedonia; ${ }^{2}$ Nephrology, Children's Clinic, Skopje, Macedonia; ${ }^{3}$ Pediatric, Private Pediatric Pratctice, Skopje, Macedonia; ${ }^{4}$ ICU, Children's Clinic, Skopje, Macedonia

\subsection{6/archdischild-2014-307384.1209}

Introduction Transient hyperphosphatemia (TH) is a benign condition, characterised by transient increase of the activity of serum alkaline phosphatase (ALKP). This condition is usually found in children under 5 years of age and elevation of ALKP activity does not last more than 4 months.

Objectives To clarify if there is evidence of bone and liver disease when activity of bone isoenzymes of ALPK is markedly elevated.

Material and methods Herein we present three infants, aged 5,12 and 18 months. All of them had febrile viral respiratory infection, which required hospitalisation. All of them had increased ALKP $637,>1000,>1000 \mathrm{U} / 1$. Liver enzymes were normal as well as the values of serum calcium and phosphorus. All children have had regular antirachitic prophylaxis. The serum ALKP normalised within 2-3 months. Increased values of ALKP were initially considered as a sign of rickets by the paediatrician who treated infants for respiratory infection and vitamin D therapy was recommended, but was not implemented after reconsideration.

Conclusion Infants with TH can be clearly identified from those having rickets by considering the age of the patient, regular vitamin D prophylaxis, history of viral infection and by excluding other causes of elevated ALKP, particularly liver or bone disease.

\section{PO-0569 PASTEURISATION OF HUMAN BREAST MILK: A NEONATAL DILEMMA}

K Stock, E Griesmaier, B Brunner, V Neubauer, U Kiechl-Kohlendorfer, R Trawöger. Pediatrics II, University Hospital, Innsbruck, Austria

\subsection{6/archdischild-2014-307384.1210}

Background Preterm infants are at risk of postnatal transmission of cytomegalovirus (CMV) via breast milk. Although most infants remain asymptomatic in the neonatal period, doubts about adverse effects on neurodevelopmental outcome have been raised. Pasteurisation prevents transmission of CMV via breast milk, which concomitantly inactivates immune and bioactive components. Data indicate that necrotizing enterocolitis (NEC) and late-onset sepsis (LOS) are less common in preterm infants fed with breast milk, as compared to infants fed with formula or pasteurised breast milk.

Aim To assess whether feeding preterm infants with unpasteurized breast milk i) decreases the rate of LOS and NEC and ii) increases the rate of postnatally acquired CMV infections.

Methods Between January 2008 and July 2013 preterm infants $<32$ weeks gestational age admitted to the neonatal intensive care unit Innsbruck $(n=341)$ were eligible for the study. Of those 323 fed with breast milk were retrospectively enrolled in the study. Two groups were formed with 164 infants being fed with unpasteurized and 159 infants with pasteurised breast milk. Results The number of infants diagnosed with postnatally acquired CMV infections was significantly higher in the non-pasteurised group as compared to the pasteurised group $(6.7 \%$ vs. $0.6 \%, p=0.006)$. There was no significant difference regarding rate of LOS $(15.9 \%$ vs. $15.1 \%, p=0.486)$ or NEC $(2.4 \%$ vs. $4.4 \%, \mathrm{p}=0.254)$.

Conclusion Feeding preterm infants with unpasteurized breast milk increases the rate of CMV infections. Of interest, we also show a non-significant trend to decreased rates of NEC, but this needs to be confirmed in larger studies.

\section{P0-0570 ZYMOSAN BUT NOT LPS, PAM3CSK4, OR FLAGELLIN INDUCES IMMUNE RESPONSES IN MONOCYTES, DCS, AND MONOCYTE-DERIVED DCS OF NEONATES COMPARABLE TO THOSE OF ADULTS}

D Tokuhara, K Nohmi, H Shintaku. Pediatrics, Osaka City University Graduate School of Medicine, Osaka, Japan

\subsection{6/archdischild-2014-307384.1211}

Increased susceptibility to infection and a tendency toward more severe outcomes than in healthy adults both illustrate the prematurity of neonatal innate immunity mediated via TLRs. However, the details of TLR-mediated neonatal innate immunity are not fully understood. Here, we investigated the differences in TLRmediated immune responses between the human neonate and adult, focusing on the cytokine profiles of monocytes, dendritic cells (DCs), and monocyte-derived DCs (MoDCs) in cord and adult blood. Purified monocytes, DCs, and MoDCs were stimulated with LPS (TLR4 ligand), Pam3CSK4 (TLR1/2 ligand), flagellin (TLR5 ligand) or zymosan (TLR2 ligand). IL-8, IL-6, and TNF concentrations were analysed in culture supernatants. Compared with the effects in adult blood, LPS-, Pam3CSK4-, and flagellin-stimulated cytokine production in cord blood was weak in monocytes, comparable in DCs, and elevated in MoDCs. In contrast, zymosan stimulation gave comparable cytokine profiles in the monocytes, DCs, and MoDCs of cord and adult blood. The immaturity of TLR-mediated innate immunity in neonates thus depends on monocytes rather than DCs. Zymosan, a cell wall extract from Saccharomyces cervisiae, is known to show vaccine adjuvant activity in adult animal, but the adjuvant activity was unclear in neonatal animal. Our results indicate that zymosan-mediated effective TLR2 signalling in neonates may be useful for developing a neonatal vaccine adjuvant.

\section{PO-0571 PATHOGENS AND EMPIRIC TREATMENT OF SEPSIS IN A NEONATAL INTENSIVE CARE UNIT}

${ }^{1} \mathrm{~S}$ Ucar, ${ }^{2} \mathrm{M}$ Firat, ${ }^{1} \mathrm{M}$ Varma, ${ }^{2} \mathrm{~T}$ Sayici. ${ }^{1}$ Department of Pediatrics, OSM Middle East Hospital, Sanliurfa, Turkey; ${ }^{2}$ Department of Infection Diseases, OSM Middle East Hospital, Sanliurfa, Turkey

\subsection{6/archdischild-2014-307384.1212}

Background and aims Sepsis is a major cause of morbidity and mortality in newborns. We aimed to assess the efficacy of the current empiric regimens commonly used in neonatal intensive care units (NICU) and to describe the characteristics of newborns with sepsis.

Methods Infants admitted to the NICU with a sepsis diagnosis during January 2012 and December 2013 were assessed in a retrospective manner. While infants with early onset neonatal sepsis (EO-NS) diagnosis had received an empiric regimen including ampicillin and gentamicin, those with a late onset neonatal sepsis (LO-NS) diagnosis received carbapenem, vancomycin and fluconazole. Culture antibiogram results were compared with empirical treatment choices. Mortality and recovery rates with empiric 